\title{
Diagnóstico clínico en el síndrome de Kabuki: fenotipo y anomalías asociadas en dos casos nuevos
}

\author{
Clinical diagnosis of Kabuki syndrome: phenotype and associated abnormalities \\ in two new cases
}

\author{
Dra. María Soledad Andersen ${ }^{a}$, Dr. Sebastián Menazzi ${ }^{a}$, Dra. Paloma Brun ${ }^{a}$, Dra. Cecilia Cocah ${ }^{a}$, \\ Dr. Giuseppe Merla ${ }^{b}$ y Dra. Andrea Solari ${ }^{a}$
}

\begin{abstract}
RESUMEN
El síndrome de Kabuki es una entidad génica caracterizada por discapacidad intelectual asociada con múltiples anomalías sistémicas.

El diagnóstico es fundamentalmente clínico y se basa en dismorfias faciales típicas, anomalías esqueléticas menores, persistencia de las almohadillas del pulpejo de los dedos y déficit de crecimiento posnatal. Otros hallazgos incluyen cardiopatía congénita, anomalías genitourinarias, fisura de paladar y/o labial, atresia anal y déficits funcionales, como mayor susceptibilidad a infecciones, enfermedades autoinmunes y endocrinológicas e hipoacusia. El objetivo de este trabajo es describir dos pacientes con diagnóstico clínico de síndrome de Kabuki, destacando los hallazgos fenotípicos y malformaciones asociadas.

Palabras clave: Kabuki, KMT2D, discapacidad intelectual, dismorfias.
\end{abstract}

\section{SUMMARY}

Kabuki syndrome is a genetic entity with multiple anomalies associated with intellectual disability. The clinical diagnosis is based on typical facial features, minor skeletal abnormalities, finger pads, and postnatal growth deficit. Other findings may include congenital heart disease, genitourinary anomalies, oral clefts, anal atresia, increased susceptibility to infections, autoimmune and endocrine disease and hearing loss. The objective of this paper is to describe two patients with clinical diagnosis of Kabuki syndrome, highlighting the phenotypic findings and associated malformations.

Key words: Kabuki syndrome, KMT2D intellectual disability, body dysmorphic disorders.

http:/ /dx.doi.org/10.5546/aap.2014.e13

a. Centro Nacional de Genética Médica Dr. Eduardo Castilla (CeNaGeM).

b. Medical Genetics Unit, IRCCS Casa Sollievo della Sofferenza Hospital, San Giovanni Rotondo, Italia.

Correspondencia:

Dra. María Soledad Andersen: solandersen@gmail.com

Conflicto de intereses: Ninguno que declarar.

Recibido: 30-7-2013.

Aceptado: 16-10-2013.

\section{INTRODUCCIÓN}

El síndrome de Kabuki (SK) fue descripto por primera vez en 1988 por Niikawa y Kuroki. Se estima la prevalencia de esta patología en $1 / 32000$ y se presenta en ambos sexos con igual frecuencia. ${ }^{1,2}$ El patrón de herencia es autosómico dominante y la mayoría corresponde a casos esporádicos, lo que sugiere que se trataría de mutaciones de novo. ${ }^{3,4} \mathrm{Si}$ bien se reportaron anomalías cromosómicas asociadas al síndrome, desde el año 2010, las mutaciones en el gen KMT2D, antes llamado MLL2 (12q12-q14), fueron identificadas como las principales causas del síndrome en pacientes afectados. ${ }^{5,6}$ Existe una variante denominada Kabuki tipo II, con mutaciones descriptas en el gen KDM6A (Xp11.3), con un fenotipo similar, pero con un patrón de herencia dominante ligado al cromosoma $\mathrm{X} .78$

El objetivo de este trabajo es describir dos pacientes con diagnóstico clínico de síndrome de Kabuki, que consultaron por discapacidad intelectual, destacando los hallazgos fenotípicos y malformaciones asociadas.

\section{Paciente 1}

Niña de 9 años sin antecedentes perinatológicos relevantes, recién nacida a término, de peso adecuado para la edad gestacional (PAEG). Presentó retraso en la adquisición de pautas motoras. En el examen físico, la antropometría fue: peso: $40 \mathrm{~kg}$, en percentilo (Pc) 90-97; talla: $130 \mathrm{~cm}$, en Pc 50-75; perímetro cefálico: $52 \mathrm{~cm}$, en Pc 50. Presentaba los signos faciales característicos (Tabla 1), estrabismo, anomalías vertebrales y escoliosis. Se realizó cariotipo en el CeNaGeM, cuyo resultado fue 46, XX [25]. La secuenciación completa del gen KMT2D en el hospital Casa Sollievo della Sofferenza, Italia, detectó la mutación c.4896_4905delAAGATGCCCTT (p.Ser1632SrfsX87) en el exón 19, consistente en una deleción que produjo un corrimiento del marco de lectura y, como consecuencia, una proteína trunca. 


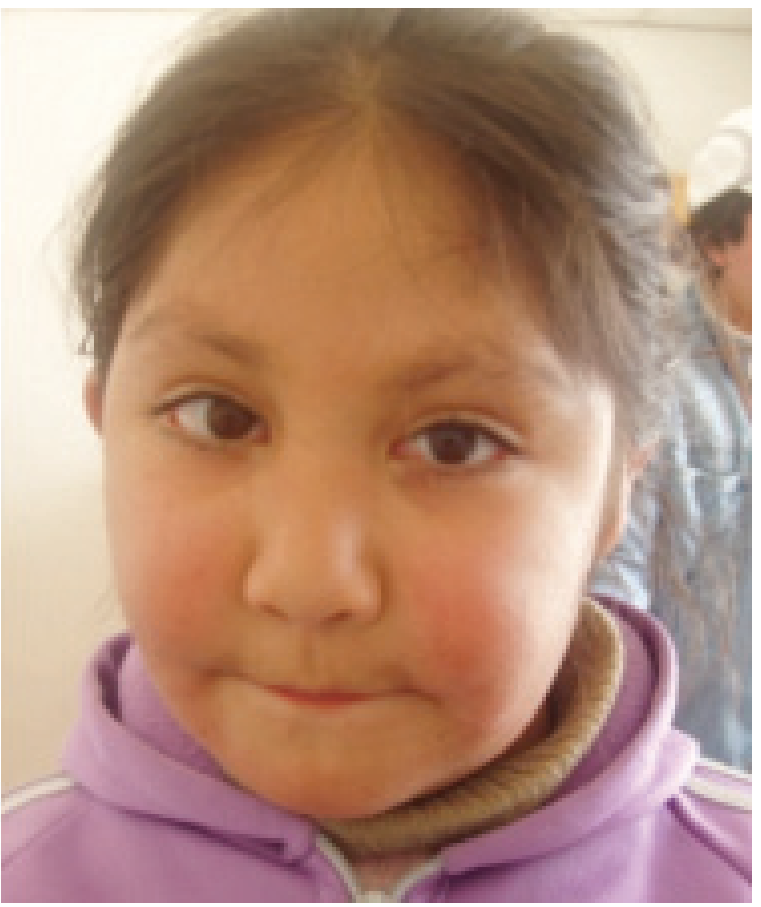

Paciente 2: niña de 5 años, nacida pretérmino a las 35 semanas por cesárea debido a hipertensión gestacional. PAEG. Alimentada por sonda nasogástrica hasta los 7 meses de vida por hipotonía, con retraso en la adquisición del lenguaje y pautas motoras. En el examen físico, la antropometría fue: peso: $17,5 \mathrm{~kg}$, en Pc 2550; talla: $107 \mathrm{~cm}$, en Pc 25-50; perímetro cefálico: $49 \mathrm{~cm}$, en Pc 25. Presentaba dismorfias típicas del síndrome (Tabla 1) y anomalías asociadas: hipoacusia derecha y estrabismo. Durante el primer año de vida, tuvo afecciones respiratorias y otitis media con repetición. Se realizó cariotipo en el CeNaGeM, y el resultado fue 46, XX [30]. No se realizó aún estudio molecular.

Las dismorfias faciales de las dos pacientes se comparan en la Tabla 1, y las anomalías asociadas, en la Tabla 2. En ambos casos, se indica la proporción de estos hallazgos en la literatura. ${ }^{1,8,9,10}$

\section{DISCUSIÓN}

Los hallazgos en las pacientes coinciden con los descriptos en la bibliografía, lo que permitió concluir que ambas presentaban SK. La ausencia de cardiopatía congénita, baja talla o anomalías urinarias en las pacientes, si bien es llamativa, no representó una dificultad para arribar al diagnóstico. El progreso de la talla y la búsqueda

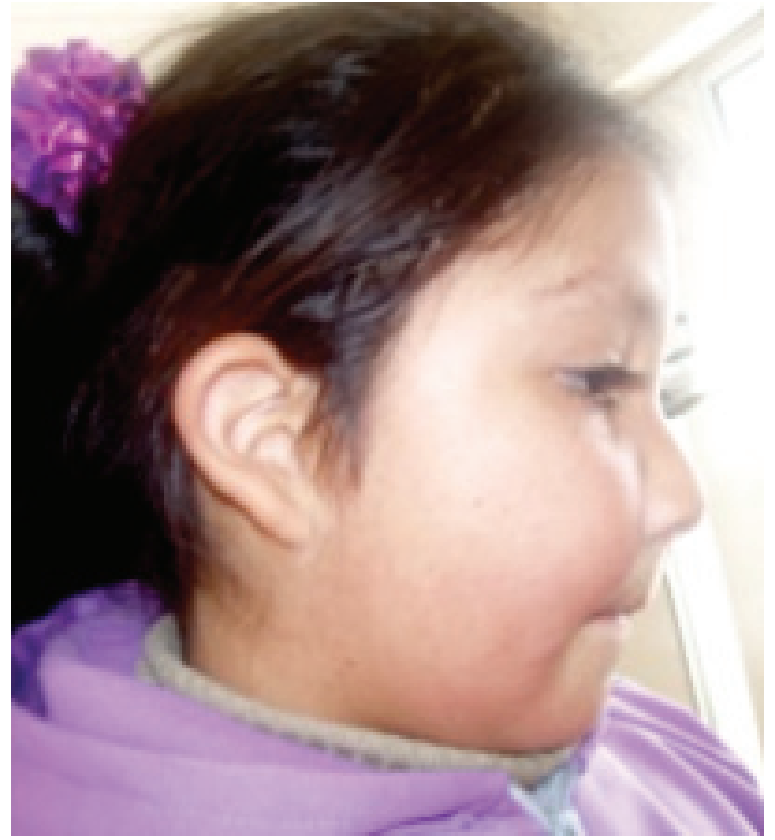

de anomalías genitales se deberían tener en cuenta en los controles futuros.

Desde el punto de vista genético, la mayoría de los casos que se reportan son de novo, por lo que el riesgo de recurrencia es bajo para la descendencia de una pareja con un hijo afectado, aunque se han descripto casos familiares con herencia dominante. ${ }^{10,11}$ Las mutaciones del gen KMT2D (en el cromosoma 12) 7,12,13 y KDM6A (en el cromosoma X) se han asociado a SK, pero debido a la existencia de casos con diagnóstico de SK, en ausencia de estas, se infiere que pueden existir otros genes involucrados.

En los casos de alta sospecha clínica o confirmación diagnóstica de síndrome de Kabuki, se recomienda el seguimiento en diferentes etapas de la vida, enfatizando el estudio y eventual manejo de los siguientes aspectos. ${ }^{1-4,14}$

- Gastrointestinal: pueden presentar dificultad en la alimentación y mal progreso de peso en los primeros meses de vida por hipotonía y reflujo gastroesofágico.

- Cardiovascular: los defectos septales auriculares y ventriculares son los más frecuentes, así como también la dilatación aórtica. Debe realizarse ecocardiograma y evaluación cardiológica a todos los pacientes.

- Lenguaje: el retraso en la adquisición del len- 

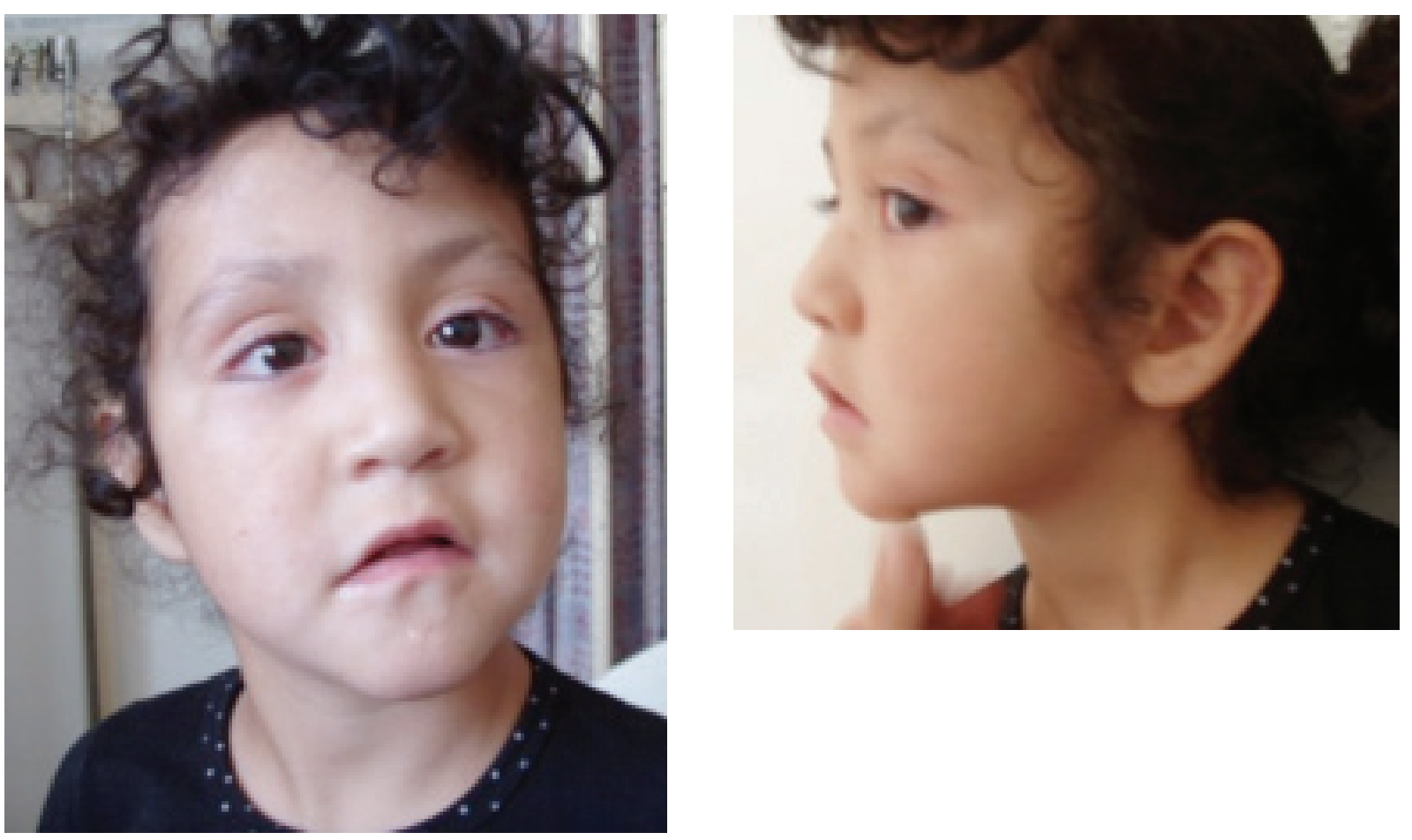

TABLA 1. Hallazgos faciales de pacientes con sindrome de Kabuki

\begin{tabular}{|c|c|c|c|c|c|c|}
\hline Características faciales & Paciente 1 & Paciente 2 & $\begin{array}{r}\text { Nikawa et al. }{ }^{1} \\
62 \text { pacientes }\end{array}$ & $\begin{array}{c}\text { Schrander-Stumpel et al. }{ }^{8} \\
29 \text { pacientes }\end{array}$ & $\begin{array}{c}\text { Sanlaville et al. }{ }^{9} \\
24 \text { pacientes }\end{array}$ & $\begin{array}{l}\text { Micale et al. }{ }^{10} \\
62 \text { pacientes } \\
\end{array}$ \\
\hline Fisuras palpebrales largas & $\mathrm{Si}^{\prime \prime}$ & $\mathrm{Si}^{\prime \prime}$ & $100 \%$ & $100 \%$ & $96 \%$ & $95 \%$ \\
\hline \multicolumn{7}{|l|}{ Eversión de tercio externo } \\
\hline del párpado inferior & Sí & Sí & $98 \%$ & $83 \%$ & $92 \%$ & $85 \%$ \\
\hline \multicolumn{7}{|l|}{ Cejas arqueadas y } \\
\hline despobladas en tercio externo & Sí & Sí & $88 \%$ & $79 \%$ & $83 \%$ & $82 \%$ \\
\hline Epicanto & Sí & No & NR & NR & NR & NR \\
\hline Estrabismo & Sí & Sí & NR & NR & $38 \%$ & $42 \%$ \\
\hline Ptosis palpebral & No & Sí & $12 \%$ & NR & $63 \%$ & $50 \%$ \\
\hline Pestañas largas & Sí & Sí & NR & NR & NR & NR \\
\hline Esclerótica azul & Sí & Sí & NR & $36 \%$ & $29 \%$ & $17 \%$ \\
\hline \multicolumn{7}{|l|}{ Labio superior fino e } \\
\hline inferior grueso & Sí & Sí & NR & NR & NR & $71 \%$ \\
\hline Paladar alto o con fisura & Sí & Sí & $79 \%$ & $69 \%$ & $63 \%$ & $60 \%$ \\
\hline Dentición anómala u oligodontia & ia Sí & Sí & $78 \%$ & $71 \%$ & $80 \%$ & $53 \%$ \\
\hline Micrognatia & No & No & NR & NR & $63 \%$ & $32 \%$ \\
\hline \multicolumn{7}{|l|}{ Puente nasal ancho con } \\
\hline punta nasal deprimida & Sí & Sí & $93 \%$ & $79 \%$ & $83 \%$ & $69 \%$ \\
\hline Orejas displásicas & Sí & Sí & $78 \%$ & $100 \%$ & $83 \%$ & $90 \%$ \\
\hline
\end{tabular}

$\mathrm{NR}=$ No reportado.

\# Fisuras palpebrales: paciente $1(3,3 \mathrm{~cm})$ y paciente $2(3 \mathrm{~cm})$, ambas por encima del Pc 95. 
TABLA 2. Anomalías asociadas en pacientes con síndrome de Kabuki

\begin{tabular}{|c|c|c|c|c|c|c|c|}
\hline Anomalías & & Paciente 1 & Paciente 2 & $\begin{array}{l}\text { Nikawa } \\
\text { et al. }{ }^{1} \\
62 \text { pac. }\end{array}$ & $\begin{array}{c}\text { Schrander- } \\
\text { Stumpel et al. }{ }^{8} \\
29 \text { pac. }\end{array}$ & $\begin{array}{c}\text { Sanlaville } \\
\text { et al. }{ }^{9} \\
24 \text { pac. }\end{array}$ & $\begin{array}{l}\text { Micale } \\
\text { et al. }^{10} \\
62 \text { pac. }\end{array}$ \\
\hline \multirow[t]{2}{*}{ Crecimiento } & Baja talla & No & No & $75 \%$ & $62 \%$ & $54 \%$ & $61 \%$ \\
\hline & Microcefalia & No & No & $5 \%$ & $31 \%$ & $48 \%$ & $29 \%$ \\
\hline \multirow[t]{3}{*}{ Miembros } & Finger Pads & Sí & Sí & $77 \%$ & $100 \%$ & $96 \%$ & $75 \%$ \\
\hline & Braquidactilia/clinodactilia & Sí & Sí & $89 \%$ & $75 \%$ & $100 \%$ & $63 \%$ \\
\hline & Hiperlaxitud articular & Sí & Sí & NR & $96 \%$ & $75 \%$ & $48 \%$ \\
\hline \multirow[t]{5}{*}{ Viscerales } & Cardiopatía & No & No & $32 \%$ & $28 \%$ & $35 \%$ & $60 \%$ \\
\hline & Urogenitales & No & No & NR & $34 \%$ & $30 \%$ & $39 \%$ \\
\hline & Hipoacusia & & & & & & \\
\hline & neurosensorial/conductiva & Sí & Sí & NR & $50 \%$ & $41 \%$ & $40 \%$ \\
\hline & Telarca precoz & No & No & NR & $50 \%$ & $39 \%$ & $16 \%$ \\
\hline \multirow[t]{4}{*}{ Neurológicas } & Epilepsia & No & No & NR & $10 \%$ & $14 \%$ & $21 \%$ \\
\hline & Hipotonía & No & Sí & NR & $83 \%$ & $79 \%$ & $60 \%$ \\
\hline & $\begin{array}{c}\text { Agenesia de } \\
\text { cuerpo calloso }\end{array}$ & No & No & NR & NR & NR & $5 \%$ \\
\hline & Déficit intelectual & Sí & Sí & Leve-moderado & Moderado & $100 \%$ & $84 \%$ \\
\hline Inmunológicas & $\begin{array}{c}\text { Enfermedad autoinmune } \\
\text { (tiroiditis) }\end{array}$ & No & No & NR & NR & NR & $7 \%$ \\
\hline Esqueléticas & $\begin{array}{c}\text { Luxación de rótula/cadera/ } \\
\text { pie plano/escoliosis }\end{array}$ & Sí & No & NR & $81 \%$ & $50 \%$ & $9,7 \%$ \\
\hline
\end{tabular}

$\mathrm{NR}=$ No reportado. Pac. $=$ pacientes.

guaje se observa en casi todos los pacientes con SK. En el caso de fisura y/o incompetencia velopalatina, deben ser remitidos a cirugía maxilofacial y rehabilitación fonoaudiológica.

- Madurativo/conductual: se describe retraso madurativo/mental y, en ocasiones, déficit de atención. El autismo ha sido reportado en pocos casos de KS.

- Neurológico: la hipotonía es frecuente. Una vez diagnosticada, debe indicarse kinesioterapia motora y estimulación temprana luego del diagnóstico. Se debe realizar resonancia magnética nuclear cerebral ya que hay malformaciones del sistema nervioso central descriptas, si bien no hay hallazgos consistentes.

- Endocrinológico: se han reportado cuadros de hipoglucemia neonatal y deficiencia de hormona de crecimiento; por esto, es importante monitorear peso y talla, dosar la hormona y considerar tratamiento sustitutivo de acuerdo con valores. Derivar a Endocrinología si clínicamente el peso y la talla están por debajo de -2 DE o bien si la curva de crecimiento decae significativamente.

Obesidad hacia la adolescencia. Recomendar ejercicio físico y seguimiento nutricional. La pubertad generalmente es normal. Puede presentarse telarca precoz.

- Traumatológico/ortopédico: hay que tener presente la laxitud articular, que conlleva la luxación congénita de caderas y rótula. La escoliosis está asociada a hipotonía y/o anomalías vertebrales. Seguimiento traumatológico y kinésico.

- Inmunológico: se deben dosar poblaciones linfocitarias e inmunoglobulinas. En caso de inmunodeficiencia, se debe derivar a Inmunología. Los pacientes presentan un riesgo incrementado de enfermedades autoinmunes, tales como púrpura trombocitopénica idiopática, 
anemia hemolítica, vitíligo y déficit de IgA.

- Aspecto auditivo: la mayoría presenta hipoacusia secundaria a otitis media crónica, pero existe un incremento de pérdida de la audición de origen neurosensorial o mixta.

- Oftalmológico: se han descripto numerosas complicaciones oculares, entre ellas, ectropión, ptosis palpebral, estrabismo, anomalías del conducto lacrimal y coloboma del iris. ${ }^{15}$

El SK es una entidad poco frecuente, pero de signos clínicos característicos. El diagnóstico temprano permite un seguimiento multidisciplinario cuya finalidad es disminuir la morbilidad, complicaciones y lograr una adecuada estimulación temprana, coordinada por el pediatra de cabecera.

\section{BIBLIOGRAFÍA}

1. Niikawa N, Kuroki Y, Kajii T, Matsuura N, et al. Kabuki make-up (Niikawa-Kuroki) Syndrome: A study of 62 patients. Am J Med Genet 1988;31:565-89.

2. Matsumoto N, Niikawa N. Kabuki make-up syndrome: a review. Am J Med Genet 2003;117C:57-65.

3. Gorlin RJ, Cohen MM Jr., Hennekam RCM. Syndromes of the head and neck. Cuarta edición. Oxford University Press 2001;21:Pág.938.

4. Jones KL. SMITH Patrones reconocibles de malformaciones humanas. Sexta edición. Madrid: Elsevier Saunders; 2007. Cap.1 C:Pág.118.

5. Ng SB, Bigham AW, Buckingham KJ, Hannibal MC, et al. Exome sequencing identifies MLL2 mutations as a cause of
Kabuki syndrome. Nat Genet 2010;42(9):790-3. doi:10.1038/ ng.646.

6. Paulussen AD, Stegmann AP, Blok MJ, et al. MLL2 mutation spectrum in 45 patients with Kabuki syndrome. Hum Mutat 2011;32:E2018-25.

7. Lederer D, Grisart B, Digilio MC, Benoit V, et al. Deletion of KDM6A, a histone demethylase interacting with MLL2, in three patients with Kabuki syndrome. Am J Hum Genet. 2012;90:119-124.

8. Miyake N, Mizuno S, Okamoto N, Ohashi H, et al. KDM6A point mutations cause Kabuki syndrome. Hum Mutat 2013;34:108-10.

9. Schrander-Stumpel C, Meinecke P, Wilson G, GillessenKaesbach G, et al. The Kabuki (Niikawa-Kuroki) syndrome: further delineation of the phenotype in 29 non-Japanese patients. Eur J Pediatr 1994;153:438-45.

10. Sanlaville D, Genevieve D, Bernardin C, Amiel J, et al. Failure to detect an 8p22-8p23.1 duplication in patients with Kabuki (Niikawa-Kuroki) syndrome. Eur J Hum Genet 2005;13:690-3.

11. Micale L, Augello B, Fusco C, Selicorni A, et al. Mutation spectrum of MLL2 in a cohort of Kabuki syndrome patients. Orphanet J Rare Dis 2011;6:38. doi: 10.1186/1750-1172-6-38. [Consulta: 9 de junio de 2011]. Disponible en PubMed.

12. Adam MP, Hudgins L. Kabuki Syndrome: a review. Clin Genet 200;67(3):209-19.

13. Suarez Guerrero JL, et al. Síndrome de Kabuki. An Pediatr (Barc) 2012. doi: 10.1016/j.anpedi.2012.01.016.

14. Philip N, Devriendt K, Clayton-Smith J. Management of Kabuki syndrome: a clinical guideline. 2010:1-23. [Created: 08/03/2010. Review Date: 08/03/2011]. Disponible en: www.dyscerne.org.

15. Turner C, Lachlan K, Amerasinghe N, Hodgkins P, et al. Kabuki syndrome: new ocular findings but no evidence of 8p22-p23.1 duplications in a clinically defined cohort. Europ J Hum Genet 2005;13:716-20. 